\title{
Cartographie De La Dynamique Du Trait De Cote A Grand-Lahou : Utilisation De L'outil « Digital Shoreline Analysis System (Dsas)"
}

\author{
Djagoua Eric M. Valère \\ Bakayoko Fatoumata \\ Kouadio Maffoué Jeanne \\ Kassi A. Jean-Baptiste \\ Mobio A. Brice Hervé
}

UFR des Sciences de la Terre et des Ressources Minières, Département de

Géosciences Marines, 22 BP 582 Abidjan 22, Côte d'Ivoire

Centre Universitaire de Recherche et d'Application en Télédétection

(CURAT), 22 BP 801 Abidjan 22, Côte d'Ivoire

\section{Adonis Krou Kouamé \\ Tiemélé Jacques André \\ Yavo Patricia}

UFR des Sciences de la Terre et des Ressources Minières, Département de

Géosciences Marines, 22 BP 582 Abidjan 22, Côte d'Ivoire

\section{doi: 10.19044/esj.2016.v12n36p327 URL:http://dx.doi.org/10.19044/esj.2016.v12n36p327}

\begin{abstract}
Like many coasts, coastal erosion is evident on all the Ivorian coast and is characterized by the destruction of physical assets (infrastructure, habitat and sightseeing). Especially in Grand-Lahou, it threatens a fishing village located on a strip of land 200 meters separating the Atlantic Ocean from the Tadjo lagoon and facing the mouth of the Bandama River. To contribute to the choice of sustainable coastal development in Grand-Lahou, this study aims to characterize the dynamics of the coastline by performing coastal erosion map locally for the best appreciation of the erosion phenomenon. To do this, the GIS which consisted of compilation of coastlines data from Landsat images from 1998, 2001, 2005, 2009, 2011 and 2014 in the study area. The dynamic of the coastline in this zone was done through the Digital Shoreline Analysis System (DSAW). The variations observed between 1998 and 2014 show a coastline fattening at an average rate of 2.09 $\mathrm{m} /$ year in the west of the river mouth before Lahou-Kpanda village and erosion with a speed of $0.84 \mathrm{~m} /$ year on rest of the shore after Lahou Kpanda village.
\end{abstract}


Keywords: Cartography, Coastal erosion, shoreline, DSAS, Côte d'Ivoire, Grand-Lahou.

\section{Résumé}

Comme c'est le cas pour la majorité des côtes, l'érosion côtière est manifeste sur la totalité du littoral ivoirien et se caractérise par la destruction de biens matériels (infrastructures, habitats et sites touristiques). Notamment à Grand-Lahou, elle menace un village de pêcheurs situé sur une bande de terre de $200 \mathrm{~m}$ séparant l'océan atlantique de la lagune Tadjo et qui fait face à l'embouchure du fleuve Bandama. En vue de contribuer aux choix d'aménagement durable du littoral à Grand-Lahou, la présente étude a pour objectif de caractériser la dynamique du trait de côte en réalisant une carte de l'érosion côtière à l'échelle locale pour une meilleure appréciation du phénomène d'érosion. Pour ce faire, l'approche SIG a consisté en une compilation de données issues des images LANDSAT de 1998, 2001, 2005, 2009, 2011 et de 2014 de la zone d'étude. La mesure de la dynamique du trait de côte dans ce secteur du littoral s'est fait par le biais du logiciel Digital Shoreline Analysis System (DSAS). Les variations observées entre 1998 et 2014 témoignent d'un littoral en engraissement à un rythme moyen de 2,09 $\mathrm{m} / \mathrm{an}$ à l'Ouest de l'embouchure avant Lahou-Kpanda et une érosion avec une vitesse de $0.84 \mathrm{~m} /$ an sur le reste du rivage après ce même village.

Mots clés : Cartographie, érosion côtière, trait de côte, DSAS, Côte d'Ivoire, Grand-Lahou.

\section{Introduction}

Comme sur la plupart des zones côtières du monde entier, les zones côtières de 1'Afrique de l'Ouest ont été marquée ces dernières décennies par une croissance démographique extrêmement importante et corrélativement par une augmentation des activités économiques sur les zones côtières. Ainsi, parallèlement à l'occupation permanente par l'urbanisation, d'autres formes d'activités mobilisent de plus en plus les espaces littoraux. Qu'il s'agisse d'activités économiques (pêches, tourisme balnéaire, agricultures), de loisirs, de vacances ou des infrastructures primordiales (routes, aéroports), le littoral demeure soumis à des pressions anthropiques chroniques. Ce point de survol entre l'homme et les côtes révèle un phénomène général de dégradation de la zone côtière, car l'urbanisation accentue les phénomènes érosifs sur les littoraux (Quensiere, 2006). Éminemment mobile, le littoral est soumis à des processus naturels (courants, houle, marée, vagues, tempêtes, ...) ou anthropiques (impact des ouvrages, opération de rechargement, ...) qui le 
conduisent à des évolutions rapides, caractérisées notamment par les notions d'aléas de submersion et d'érosion (Mallet et al., 2012).

À l'interface entre terre et mer, le littoral est un milieu fragile, très convoité, qu'il est important de connaître afin de le gérer au mieux.

La zone côtière ivoirienne qui fait partir de la zone côtière de l'Afrique de l'Ouest, est sujette à érosion marine. En effet, les 2/3 du trait de côte ivoirien sont déstabilisés par l'érosion côtière, et ne cesseront de croître vu l'amplification de la pression socio-économique sur le littoral et les projections mondiales relatives aux impacts des changements climatiques laissant envisager une accentuation de l'élévation du niveau de la mer, avec pour corollaire un renforcement des processus d'érosion sur les côtes basses (Hauhouot, 2000).

L'érosion du littoral concerne toutes les côtes orientales ivoiriennes entre Fresco et Assinie. Cinq points chauds ont été identifiés le long du littoral ivoirien à savoir San-Pedro, Port-Bouet, Grand-Bassam, Assinie et GrandLahou. Ces villes côtières ont la particularité de présenter un enjeu économique pour la Côte d'Ivoire du fait qu'elles abritent les grandes infrastructures économiques et accueillent la majorité de la production nationale de pêche maritime. Au niveau de Grand-Lahou qui est le plus important secteur de pêche, l'érosion côtière y menace Lahou Kpanda, un village de pêcheur et se caractérise par la destruction des infrastructures, des sites touristiques et des habitats.

$\mathrm{Vu}$ le réel danger que présente l'érosion côtière pour ce village, et devant le besoin de prévenir les risques côtiers dans un contexte de changement climatique, il est primordial de caractériser le phénomène d'érosion à Grand-Lahou. C'est la raison essentielle de l'étude de la dynamique du trait de côte. Le suivi et l'analyse des indicateurs de l'évolution côtière sont des préalables à la gestion du littoral (Mallet et al., 2012).

\section{Présentation de la zone d'étude}

La Côte d'Ivoire est subdivisée en deux entités géologiques d'importance inégales, chronologiquement distinctes (Tagini, 1971). Il existe d'une part un bassin sédimentaire d'âge fin secondaire à quaternaire au Sud qui présente 2,5 pourcent du territoire et est caractérisé par des systèmes lagunaires et d'autre part, un socle précambrien qui occupe les 97,5 pourcent restant.

La zone d'étude est une petite partie du littoral de la Côte d'Ivoire, plus précisément de Grand-Lahou. Le littoral de Grand-Lahou est situé entre les latitudes $5^{\circ} 12^{\prime} \mathrm{N}$ et $5^{\circ} 9^{\prime} \mathrm{N}$ et les longitudes $4^{\circ} 56 \mathrm{~W}$ et $5^{\circ} 70 \mathrm{~W}$ (figure 1 ). 


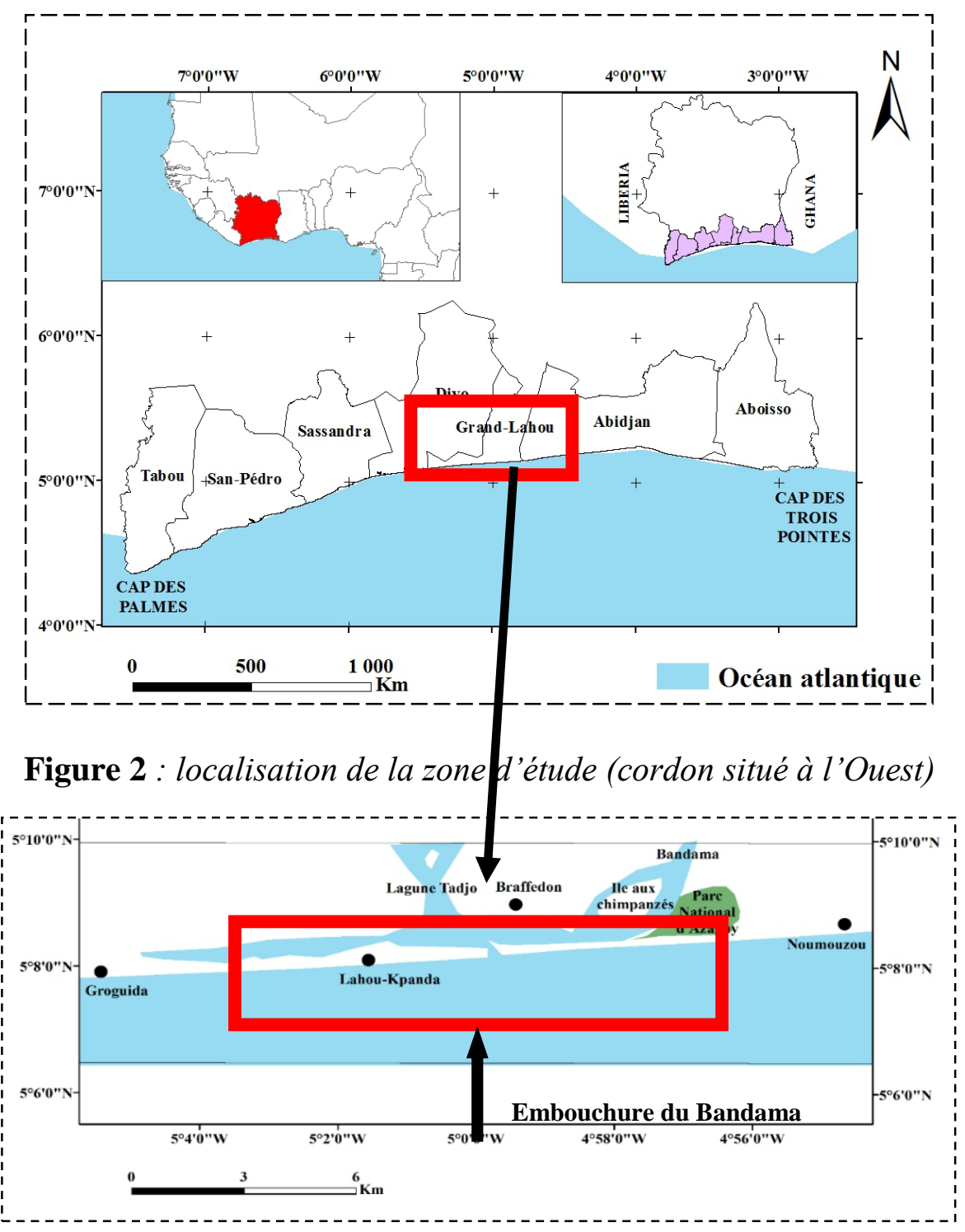

\section{Méthodologie}

Dans le cadre de cette étude, nous avons utilisé des images satellitaires LANDSAT TM et ETM+. Il s'agit d'une (01) image TM de 1998 et de cinq (05) images ETM+ de 2001, 2005, 2009, 2011 et 2014. Ces images sont de la scène (Path/Row) 196-056 avec une résolution de 28,5 m. Le traitement des images s'est déroulé en trois étapes en tenant compte des logiciels utilisés. D'abord dans le logiciel ENVI 5.1, nous avons effectué un géo référencement afin de rendre les différentes images superposables. Ensuite un seuillage a permis d'identifier grâce à une limite nette (ligne de référence) les populations de pixels représentant la zone terrestre (pixels clairs) et le domaine marin 
(pixels sombres). Ainsi la ligne de référence mise en évidence est l'ados. Le logiciel ArcGIS Desktop 10.2.2 a été utilisé pour la digitalisation du trait de côte et la réalisation des cartes. Enfin DSAS v4.3, une extension, a permis le calcul statistique des vitesses d'érosion (EPR) et des taux d'évolution (LRR) à partir des différents traits de côte extraits des images utilisées. Cet outil a pour avantage de donner une évolution graphique et statistique du trait de côte. Il fournit une méthode normalisée qu'on peut répéter pour le calcul de l'évolution statistique tout en analysant le changement passé, présent et futur du littoral. L'utilisation du logiciel DSAS nécessite au préalable la préparation des traits de côte au format vecteur au sein d'une géodatabase dans ArcCatalog et la numérisation d'une ligne de base imaginaire à partir de laquelle DSAS crée des transects qui viendront couper les différents traits de côtes. Pour cette étude, nous avons utilisé comme pas de mesures $10 \mathrm{~m}$ entre les transects long de $200 \mathrm{~m}$. Le maillage fin $(10 \mathrm{~m})$ permet de mieux apprécier l'évolution du littoral dans le détail et préciser le comportement du littoral localement.

\section{Résultats et interprétation}

L'étude de la dynamique du trait de côte avec l'extension DSAS 4.3 a montré soit une érosion soit un engraissement en fonction de l'année étudiée. D'après l'analyse des différentes cartes réalisées, l'évolution graphique du trait de côte montre une nette variation par secteur et par année. La synthèse de ces cartes montre de 1998 à 2014 un engraissement sur le rivage situé à 1'Ouest de Lahou-Kpanda à Groguida et à 1'Est une érosion. La valeur négative matérialise une érosion et la valeur positive un engraissement.

\section{évolution graphique du trait de côte}

Le tableau 1 présente la dynamique du trait de côte au niveau de Groguida. D'après le tableau, au niveau du secteur de Groguida, la zone est identifiée en moyenne comme une zone d'engraissement avec une vitesse de 2,09 m/an. Cette zone est marquée par une érosion entre 1998-2001 et 20052009 avec des vitesses respective de $-2,56 \mathrm{~m} / \mathrm{an}$ et $-1,55 \mathrm{~m} / \mathrm{an}$, tandis que de 2001 à 2005, 2009 à 2011 et de 2011 à 2014, elle a subit un engraissement en moyenne de $3,52 \mathrm{~m} / \mathrm{an}$.

\begin{tabular}{|c|c|c|}
\hline Années & Phénomène & Vitesse d'érosion en m/an \\
\hline $1998-2001$ & érosion & $-2,56$ \\
\hline $2001-2005$ & Engraissement & $+3,54$ \\
\hline $2005-2009$ & érosion & $-1,55$ \\
\hline $2009-2011$ & Engraissement & $+3,34$ \\
\hline $2011-2014$ & Engraissement & $+3,68$ \\
\hline $1998-2014$ & Engraissement & $+2,09$ \\
\hline
\end{tabular}

Tableau 1 : synthèse de l'évolution du trait de côte à Groguida 
Le tableau 2 quant à lui présente la dynamique du trait de côte au niveau de Lahou-Kpanda. Le phénomène dominant dans ce secteur est l'érosion avec une vitesse de $-0,84 \mathrm{~m} / \mathrm{an}$. Durant la période d'étude, nous avons observé une inversion des tendances. De 1998 à 2011, la zone a subit une érosion avec une vitesse moyenne de $-1,66 \mathrm{~m} / \mathrm{an}$ et un engraissement de $+2,43 \mathrm{~m} / \mathrm{an}$. Cependant on note de petite variation au niveau du village de Lahou-Kpanda.

En effet, l'érosion de 1998 à 2001 et 2005 à 2009 a commencé à environ $1 \mathrm{~km}$ avant Lahou-Kpanda pour s'étendre au-delà.

De 2001 à 2005, elle subit un engraissement qui commence à LahouKpanda et s'étend jusqu'à $1 \mathrm{~km}$ après. Mais, après les $1 \mathrm{~km}$, plus proche de l'embouchure, c'est l'érosion qui est observé.

De 2009 à 2011, l'engraissement est continue de Lahou-Kpanda jusqu'à $950 \mathrm{~m}$ après, au-delà de cette limite, on a une érosion jusqu'à l'embouchure. Cela démontre que les terres plus proches de l'embouchure sont en constant érosion. Ce fait est dû à la migration de l'embouchure du fleuve Bandama qui se fait de l'Est vers l'Ouest.

\begin{tabular}{|c|c|c|}
\hline Années & Phénomène & Vitesse d'érosion (m/an) \\
\hline $1998-2001$ & Erosion & $-2,47$ \\
\hline $2001-2005$ & Erosion & $-1,62$ \\
\hline $2005-2009$ & Erosion & $-0,64$ \\
\hline $2009-2011$ & Erosion & $-1,91$ \\
\hline $2011-2014$ & Engraissement & $+2,43$ \\
\hline $1998-2014$ & Erosion & $-0,84$ \\
\hline
\end{tabular}

Tableau 2 : synthèse de l'évolution du trait de côte à Lahou-Kpanda

\section{Dynamique du trait de côte dans le logiciel DSAS}

La figure 2 est un Modèle cartographique qui monte l'évolution des vitesses d'érosion et d'engraissement basée sur la méthode des points extrêmes (EPR). De l'Ouest vers l'Est de la zone d'étude, la figure permet d'identifier quatre (04) secteurs caractérisés par un phénomène d'engraissement. Les trois premiers secteurs présentent des vitesses d'engraissement constantes avec une vitesse moyenne de $3,20 \mathrm{~m} / \mathrm{an}$. On peut les observer à partir des transects cumulés 132, 337 et 739 avec des vitesses d'engraissement respectivement de 3,$12 ; 3,12$ et 3,36 m/an. Le dernier et quatrième secteur matérialisé par 1182 transects cumulés présente une vitesse d'engraissement moyenne de $2,43 \mathrm{~m}$ /an avec une grande variation de la vitesse qui part de $5,05 \mathrm{~m} / \mathrm{an}$ à $1,75 \mathrm{~m} / \mathrm{an}$.

La figure 3 quant à elle est un modèle cartographique qui montre l'évolution du taux d'érosion et d'engraissement basée sur la méthode de régression linéaire (LRR). Elle permet d'identifier deux grands secteurs. Le premier secteur matérialisé par 1107 transects cumulés est caractérisé par un phénomène d'engraissement avec une vitesse 2,25 m/an. Le second secteur 
caractérisé par un phénomène d'érosion est matérialisé 1182 transects cumulés avec une vitesse d'érosion égale $-0,84 \mathrm{~m} / \mathrm{an}$.

Figure 3: Evolution des vitesses d'érosion et d'engraissement basée sur la méthode des points extrêmes (EPR)
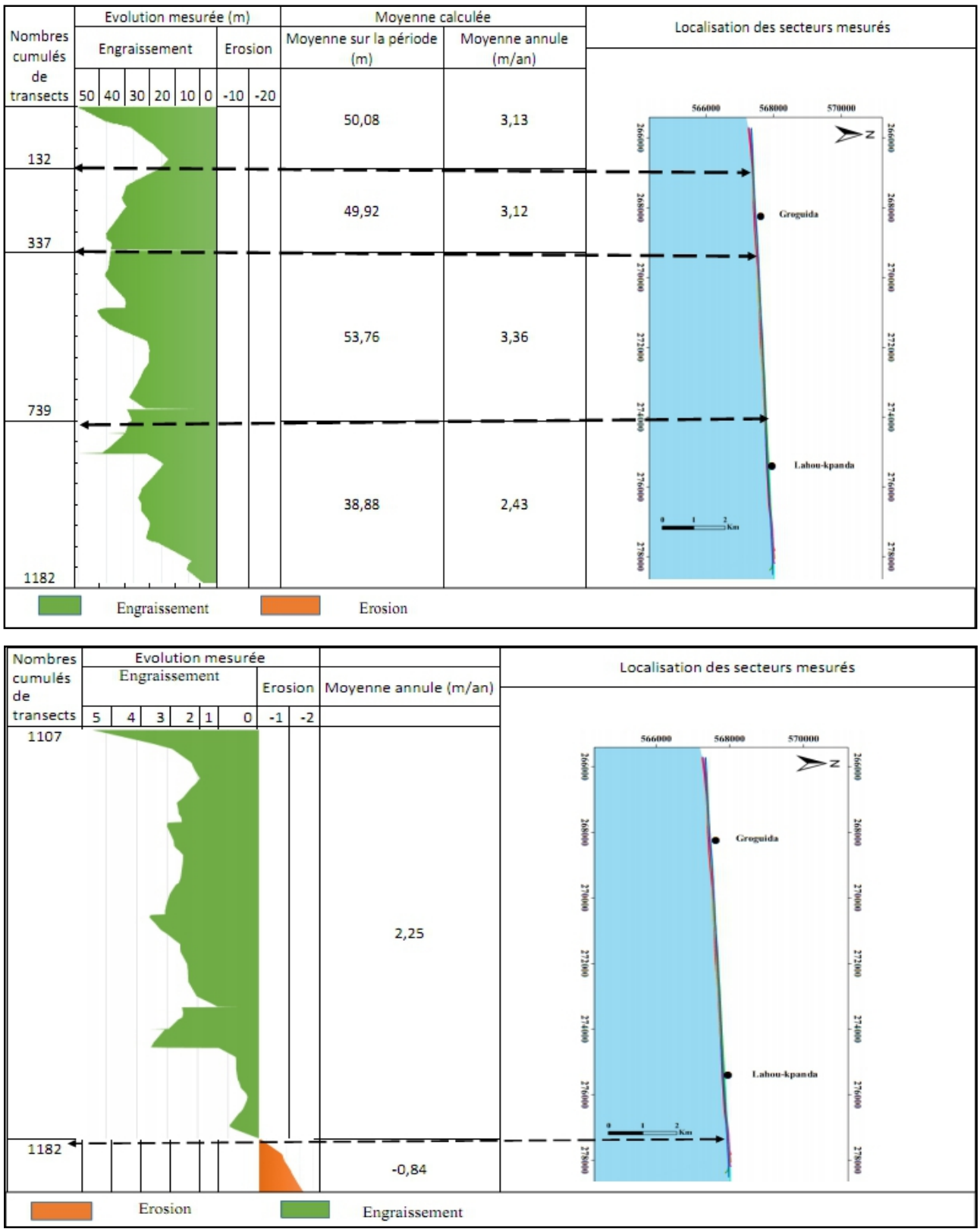

Figure 4: Evolution du taux d'érosion et d'engraissement basée sur la méthode de

\section{Discussion}

régression linéaire (LRR)

Dans le cadre de cette étude, nous avons utilisé la méthode des points extrêmes et la méthode de la régression linéaire proposées par le logiciel 
DSAS. Nous avons pu constater que des deux méthodes, la plus efficace est la méthode de la régression linéaire car, elle tient compte de toutes les positions du trait de côte, contrairement à la méthode des points extrêmes qui néglige le détail entre les deux dates choisies.

Nos résultats présentent certaines différences avec les études précédentes notamment celle réalisé par Hauhouot et al.,1997 ; Abe, 2005 et Koffi et al 1987. Alors que tous parlent de recul du trait de côte, la moyenne des vitesses d'érosion entre 1998 et 2014 fait état d'un engraissement de l'ordre de 2, $09 \mathrm{~m} /$ an à l'Ouest de l'embouchure, bien que l'on observe une alternance entre les phénomènes d'érosion et d'engraissement. A partir de Lahou-Kpanda, notre résultat montre une érosion avec un taux moyen de 0,84 $\mathrm{m} / \mathrm{an}$. Cela pourra être dû au fait qu'il est situé trop près de l'embouchure du fleuve Bandama. En effet, cette partie subit une érosion latérale du rivage provoqué par l'océan atlantique et une érosion frontale dûe à la migration de 1'embouchure. Ces résultats sont semblables à ceux obtenus par Hauhouot et al., (1997) sur la cinématique du littoral de Grand-Lahou. Il a révélé que l'érosion s'est faite au rythme mesuré de 0,3 m/an entre 1957 et 1986 avec une accélération de $2 \mathrm{~m}$ /an sur la période de 1986-1993. En période d'étiage, on observe au niveau de l'embouchure, des rubans de sédiment qui stagnent et sont piégés dans le fleuve, incapable de se déverser dans la mer pour combler le déficit de sédiments causé par le transit sédimentaire. Ce phénomène d'érosion normalement considéré comme un aléa naturel serait amplifié par la construction sur le fleuve Bandama de deux barrages hydroélectriques en amont : Kossou en 1972 et Taabo en 1980. En l'absence de toute perturbation anthropique, les plages ont-elles une capacité de résilience naturelle. Pourtant, ces barrage bloquent en partie les sédiments et freinent ainsi la reconstitution du littoral attaqué par l'océan. Malgré cela, nous avons pu observer une alternance entre l'érosion et l'engraissement. Il a été observé sur la période de 1985 à 1987, un recul de 10 mètres du littoral, soit une érosion de 3 m/an par Koffi et al., 1987. Quant à Abe (2005), au cours de ses travaux couvrant la période 1950 à 1995 a estimé qu'à GrandLahou la vitesse de recul était $2 \mathrm{~m} / \mathrm{an}$.

\section{Conclusion}

L'étude réalisé en l'utilisation le logiciel « Digital Shoreline Analysis System » a contribué à la cartographie de la dynamique de trait de côte à Grand-Lahou et à la connaissance du phénomène d'érosion à Grand-Lahou. L'objectif principal est de réaliser une carte locale de l'érosion pour une meilleure prise de décisions quant aux aménagements côtiers pour la protection des biens et des personnes.

Il ressort que le littoral ivoirien à Grand-Lahou est fragile et présente des zones instables et des zones plus ou moins stables. La tendance évolutive 
montre une avancée du niveau de mer avec une vitesse de 2,09 m/an pour le trait de côte proprement dite et une érosion au niveau même de l'embouchure avec une vitesse de $86,60 \mathrm{~m} / \mathrm{an}$. La capacité de résilience des plages fait qu'il y’a une alternance entre érosion et engraissement permettant de rééquilibrer autant que possible la plage. Cependant, avec ce recul spectaculaire de l'embouchure, il y a lieu de faire une évaluation rigoureuse et une surveillance périodique de l'évolution du trait de côte à petite échelle dans ce secteur en tenant compte de tous les aspect liée à la dynamique du littoral notamment la pression anthropique sur le littoral afin réaliser une carte de sensibilité à l'érosion du littoral qui devrait aider à l' aménagement de ce secteur.

\section{References:}

1. Abe J. (2005). Contribution à la connaissance de la morphologie et de la dynamique sédimentaire du littoral ivoirien (cas du littoral d'Abidjan) : essai de modélisation en vue d'une gestion rationnelle. Thèse de Doctorat d'Etat, Université de Cocody, $197 \mathrm{p}$

2. Hauhouot C. (2000). Analyse et cartographie de la dynamique du littoral et des risques « naturels » côtiers en Côte d'Ivoire. Thèse de doctorat, Université de Nantes, UFR de géographie et d'Aménagement Régional UMR 6554 CNRS, 289 p.

3. Hauhouot C., Robin M. et Miosec A. (1997). Cinématique du littoral de Grand-Lahou à l'aide d'un SIG, In : îles et littoraux tropicauxBrest, Ouest éditions, pp 269-276v

4. Koffi K. P., ABE J.et Affian K. (1987). Evolution actuelle du littoral ivoirien. Résultats préliminaires du suivi 1985-1987. Centre de Recherche d'Océanographie. Abidjan, $7 \mathrm{p}$

5. Mallet C., Michot A. (2012). Synthèse de référence des techniques de suivi du trait de

6. côte - Rapport BRGM/RP-60616-FR, 162 p.Quensiere J., Diaw A. T., Ould El Moustapha Senhoury A., Charles-Dominique E. (2006). Un réseau ouest - africain de recherche sur la gestion durable des zones côtières : le projet "Bilan Prospectif", dans Symoens (J.-J.) (dir.), Les écosystèmes côtiers de l'Afrique de l'Ouest. Diversité biologique - Ressources- Conservation, Bruxelles, SBWOA, p. 159176

7. Tagini B. (1971). Esquisse structurale de la Côte d'Ivoire. Essai de géotechnique régionale. SODEMI Abidjan, 302 p 